\title{
Publisher's Note: Phenomenological profile of top squarks from natural supersymmetry at the LHC [Phys. Rev. D 95, 055012 (2017)]
}

Howard Baer®, Vernon Barger, Natsumi Nagata, and Michael Savoy

(Q) (Received 17 March 2021; published 29 March 2021)

DOI: 10.1103/PhysRevD.103.059902

This paper was published online on 14 March 2017 with an error in the author list. The last author's name should read as "Michael Savoy". The author's name has been corrected as of 19 March 2021. The author's name is incorrect in the printed version of the journal. 